\title{
Parametrical investigation for the optimization of spherical jet-stirred reactors design using large eddy simulations
}

\author{
Ghazaleh Esmaeelzade $^{1} \cdot$ Kai Moshammer $^{1} \cdot$ Detlev Markus $^{1} \cdot$ Ulrich Maas $^{2} \cdot$ Holger Grosshans $^{1}$
}

Received: 11 December 2020 / Accepted: 20 July 2021

Published online: 04 August 2021

(c) The Author(s) 2021

OPEN

\begin{abstract}
Due to the importance of gas-phase chemical reaction kinetics in low-emission combustion, stirred tank reactors have been used for decades as an experimental tool to study high- and low-temperature oxidation. A Jet-Stirred Reactor (JSR) setup is valuable to determine the evolution of species mole fractions. For the accuracy of the experimental results, it is important that a JSR is designed such that the concentration field is as homogeneous as possible in order to avoid disturbance of the chemical kinetics. In this work, numerical simulations were performed to investigate the mixing in a JSR chamber. The turbulent structures inside the JSR and the nozzles are captured using Large Eddy Simulations. We conducted numerically a parametric study to evaluate the effects of thermodynamic conditions and geometrical parameters on the mixing characteristics. More specifically, the diameter of the spherical chamber is modified together with the diameter of the nozzles through which fresh gases are fed. The characterization of the gas flow inside a typical spherical JSR layout and results derived by the normalized standard deviation of a tracer mass fraction show that a reduction of the JSR diameter at high pressures improves the homogeneity. Further, we propose a new optimized configuration consisting of six nozzles pointing to the center of the reactor which provides a more uniform composition compared to the standard JSR design.
\end{abstract}

Keywords Parametric study · Computational fluid dynamic · Jet stirred reactor $\cdot$ Large eddy simulations

\section{Introduction}

In the last decades, a high demand for clean combustion and a high energy conversion efficiency emerged to meet the stringent environmental standards on pollutant emissions. To this end, the chemical kinetics of alternative/renewable fuels have been extensively investigated through lab-scaled model experiments, like flames or chemical reactors $[1,2]$. The development of the kinetic mechanisms is supported by measurements in continuous and batch reactors [3]. Accordingly, validation and developing extensive chemical reaction mechanisms in such systems reduce total costs and efforts in engine manufactures. A Jet-Stirred Reactor (JSR) as an ideal continuous stirred tank reactor is particularly suitable for studies of gas-phase kinetics. To analyze the chemical reactions in detail, JSRs are usually coupled with diagnostic tools. The homogeneity of the spherical JSRs under different experimental conditions is the main requirement to investigate the simple 0-D kinetic modeling of this reactor type. Therefore, enhancing the homogeneity of the composition is achieved by chaotic advection through stirring, provided

Supplementary Information The online version contains supplementary material available at https://doi.org/10.1007/s42452-021-04743w.

$\triangle$ Ghazaleh Esmaeelzade, ghazaleh.esmaeelzade@ptb.de | ${ }^{1}$ Physikalisch-Technische Bundesanstalt, Braunschweig, Germany. ${ }^{2}$ Karlsruhe Institute of Technology, Karlsruhe, Germany.

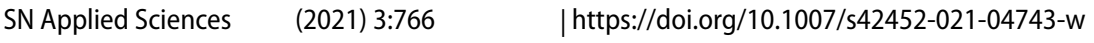


by turbulent jets inside the spherical JSR. In fact, the mixing rate and, thus, the realized level of homogeneity is the key factor to obtain chemical kinetic mechanisms of an optimized accuracy. Furthermore, simulations like Computational Fluid Dynamics (CFD) coupled with a developed kinetic mechanism are excellent for providing a general model that can be applied in several applications; consequently, this reduces the expense of experiments.

In a JSR experiment, premixed reactants are introduced into the reactor by a number of nozzles and well-mixed products are continuously removed at the outlet. These nozzles can be of different designs, e.g., inclined, crossed, or disk shape. Also, JSRs have so far been operated in various pressure and temperature ranges [4]. Davani and Ronney [5] even proposed a configuration of eight jets, each surrounded by a concentric outlet aiming to improve the mixing and consequently experimental accuracy. Later, the jet arrangement was improved by Zhang et al. [6] by adding four holes on the reactor wall to remove the exhaust gas. They concluded that the design of an inwardly offcenter shearing chamber generates small vortices over the chamber. These vortices promote the reactor performance in terms of mixture uniformity and the distribution of species compared to a traditional JSR. Esmaeelzade et al. [7] studied the mixing and flow field characteristic inside a JSR with four inclined nozzle through highly-resolved CFD analysis. Their results agreed well with the experimental data from the literature [8], especially for lower flow rates. Further, De Oliveria et al. [9] simulated an enhanced JSR configuration by considering a hemispherical premixed chamber and an outlet pipe close to the equatorial plane of the reactor. They reported that the improved geometry results in an increase of turbulence, mixing efficiency, and homogeneity of the temperature profile. All these discussed studies demonstrate the potential to enhance the mixing quality dependent on the geometrical configuration of the reactor.

Typically, the experimental setup consists of an electrically heated oven and a pressure-resistant jacket which completely enclose the JSR. Due to these enclosures only a limited optical accessibility to the flow field is given and detailed experimental information concerning the complex interaction of the jets is not available.

MacMullin and Weber [10] first proposed to characterize the macro-mixing inside real chemical reactors compared to ideal ones based on their Residence Time Distributions (RTD). The RTD is determined in general by injecting a tracer into the reactor and then monitoring the purged tracer concentration in the effluent stream as a function of time [3]. Wheres the RTD is an integral quantity, a detailed picture of the condition in the reactor is still lacking. Thus, the need to gain deeper insight in the mixing in a JSR motivates CFD simulations. A more detailed understanding of the flow field characteristic would enable to facilitate further improvement of the JSR design $[11,12]$ which would finally lead to the more accurate characterization of chemical mechanisms.

In the context of our study, we analyze the flow generated inside the JSR by means of unsteady three-dimensional LES. Based on these data we attempt to identify flow patterns such as dead zones and short circulations which are responsible for the non-homogeneity inside JSRs. The results of our simulations are compared to the RTD data of the previous experiments by Ayass et al. [8] and CFD analysis by the authors of the present paper [7]. Our results indicate that some of the commonly used reactors could have significant concentration non-homogeneity and therefore may not be suitable for chemical kinetic studies. Further, we perform a parametric study to optimize the JSR in terms of the diameters of the nozzles and the chamber, and the temperature and pressure. Finally, we suggest a new JSR design including six impinging jets which provides a more efficient mixing compared to the typical JSR configuration of four jets.

\section{Mathematical model and numerical setup}

In this section, the details of the underlying mathematical model, numerical methods, the studied JSR geometry, and the employed grid resolution are described.

\subsection{Applied mathematical model}

The numerical simulations were performed using the open source software package OpenFOAM [13]. To solve numerically the transient, turbulent flow of a compressible non-reacting mixture, we have used the reactingFOAM solver. Therein, the Favre-filtered form of mass, momentum, energy conservation for Newtonian fluids, species transport equation for each involved species together with the equation of state for ideal gases are solved in Eulerian framework given by [14]

$\frac{\partial \bar{\rho}}{\partial t}+\nabla \cdot(\bar{\rho} \widetilde{\boldsymbol{u}})=0$,

$\frac{\partial(\bar{\rho} \widetilde{\mathbf{u}})}{\partial t}+\nabla \cdot(\bar{\rho} \widetilde{\mathbf{u}} \otimes \widetilde{\mathbf{u}})=-\nabla \cdot(\bar{\rho} \mathbf{I})$
$\quad+\nabla \cdot\left[\bar{\rho} v_{\text {eff }}\left((\nabla \otimes \widetilde{\boldsymbol{u}})+(\nabla \otimes \widetilde{\boldsymbol{u}})^{T}-\frac{2}{3}(\nabla \cdot \widetilde{\boldsymbol{u}}) \mathbf{I}\right)\right]$,

$\frac{\partial \bar{\rho} \widetilde{Y}_{k}}{\partial t}+\nabla \cdot\left(\bar{\rho} \widetilde{\boldsymbol{u}} \widetilde{Y}_{k}\right)=\nabla \cdot\left(\bar{\rho} D_{k} \nabla \widetilde{Y}_{k}\right)+\overline{\dot{\omega}_{k}}$,

where the symbol ${ }^{-}$is used for Favre-filtered variables in LES; the $\sim$ is applied to the filtered density-weighted variables. In the above equations, velocity $\boldsymbol{u}$ and mass fraction $Y_{k}$ are functions of both space and time, $t$. In addition, 
density, effective kinematic viscosity, pressure, effective mass diffusivity of the species $k$ and identity matrix are denoted by $\rho, v_{\text {eff }}, p, D_{k}$ and $I$ respectively. On the right hand side of Eq. (3) the source term $\dot{\omega}_{k}$ represents the source terms of species recombination during chemical reactions. Since we consider a non-reacting flow, in the present study these terms are neglected. Furthermore, the pressure-velocity coupling is achieved using the PIMPLE algorithm which is a hybrid between PISO [15] and SIMPLE [16]. We chose PIMPLE loop in order to increase the Courant number limitation and to ensure the simulation stability.

In the present study, the solver uses a thermophysical model based on the compressibility of the fluid for a system with a fixed composition. Moreover, the specific heat capacity of pure gas is obtained by the JANAF thermochemical tables [17]. Therefore, the thermophysical properties of the mixture are calculated by the mixing law [18].

Moreover, the governing equations are discretized using the FVM method. Since the generation of a fine grid compensates the necessity of the higher order discretization, a first order approximation is applied for time advancement and second order for all spatial derivatives.

The linear equation system for the pressure is solved using the PCG (Preconditioned Conjugate Gradient) method and the preconditioned DIC (Diagonal-based Incomplete Cholesky) in which the reciprocal of the preconditioned diagonal and the upper coefficients divided by the diagonal are calculated and stored [19]. The absolute tolerance for pressure was set to $10^{-6}$ and the relative tolerance to 0 . Furthermore, the PBiCG (Preconditioned $\mathrm{Bi}$-conjugate Gradient) solver together with the pre-conditioner DILU (Diagonal-based Incomplete LU) are chosen for the velocity and turbulent kinetic energy. The absolute tolerances of the mentioned variables are equal to $10^{-6}$ and the relative tolerances to 0 .

Turbulent flows are characterized by a wide range of length and time scales. The kinetic energy in the flow transfers from large to the small scales, eventually being dissipated at the smallest scale. The large scales are most energetic and transport of the momentum and other conserved fluid properties are mostly carried out by these scales. Because of that, in the LES philosophy large scale motions are solved directly on the grid and small scales are modeled. In the filtering procedure of the LES, each variable is decomposed into a filtered component which will be resolved by Eqs. 1, 2, 3 and a sub-grid component. Therefore, the effective kinematic viscosity is the sum of the molecular viscosity $v$ and the residual or Sub-Grid Scale (SGS) eddy viscosity $v_{\text {sgs }}$. In this study, to reduce the computational costs and achieve a reasonable accuracy, we have chosen the standard Smagorinsky model. Thus, residual (sub-filter) scale stresses are accounted for via the Smagorinsky model [20], where the SGS viscosity is defined as

$v_{\text {sgs }}=\left(C_{s} \Delta\right)^{2}|\tilde{S}|$,

where $|\tilde{S}|, \Delta$, and $C_{\mathrm{s}}$ represent the filtered magnitude of the rate of strain tensor, the characteristic size of the filter, and the static Smagorinsky model coefficient, respectively. The classical Smagorinsky constant is calculated as $C_{s}=C_{k} \sqrt{C_{k} / C_{\epsilon}}$ where the default constants of the Smagorinsky model implementation in OpenFOAM $C_{k}$ and $C_{\epsilon}$, are 0.094 and 1.048 respectively [21]. Having the dynamic viscosity of the pure gases available based on Sutherland's law, the mixture viscosity of $\mathrm{CO}_{2}-\mathrm{N}_{2}$ composition is calculated according to the mixing laws [18].

Likewise, the effective mass diffusivity consists of the resolved mass diffusivity $v / S c$ and the sub-grid scale turbulent mass diffusivity $v_{t} / S c_{t}$ where the turbulent $S c h m i d t$ number $S c_{t}$ is assumed to be equal to unity.

The fluid elements entering the JSR continuously withdraw from the reactor and the time which various elements stay inside can be different. Some molecules may remain in the reactor indefinite while others almost immediately leave the reactor. The average time which the elements have spent within the boundaries of the reactor is defined as mean residence time, $\tau=V / Q$, where $V$ is the reactor volume, and $Q$ the volumetric flow rate [1]. Numerical calculation of residence time is based on the tracer concentration function $C(t)$ as follows $[3,22]$

$\frac{C_{\text {out }}(t)}{C_{0}}=\mathrm{e}^{-t / \tau}$.

Equation 5 relies on the exponential decay of the tracer concentration leaving the outlet $C_{\text {out }}$ at the time, $t$, which is related to the initial steady state concentration of the tracer $C_{0}$. It is calculated based on the tracer concentration at $t$ divided by the area under the concentration curve.

\subsection{Geometry and conditions of the parametric study}

The base configuration of the JSR in this study follows the design of a spherical reactor with four inclined nozzles proposed by Dagaut et al. [11]. The exemplary computational domain illustrated in Fig. 1 is composed of a spherical chamber of a diameter of $40 \mathrm{~mm}$ with four nozzles each of a diameter of $1 \mathrm{~mm}$ in the equatorial plane. The upward and downward pointing nozzles together with the nozzles pointing horizontal generate two circulating streams, parallel to the $x-z$ and $x-y$ planes, respectively (see the arrows at the nozzles' outlet in Fig. 1). The outlet of a diameter of $10 \mathrm{~mm}$ is placed in the surface parallel to the $x-y$ plane. The internal field of this geometry was meshed. The generated 


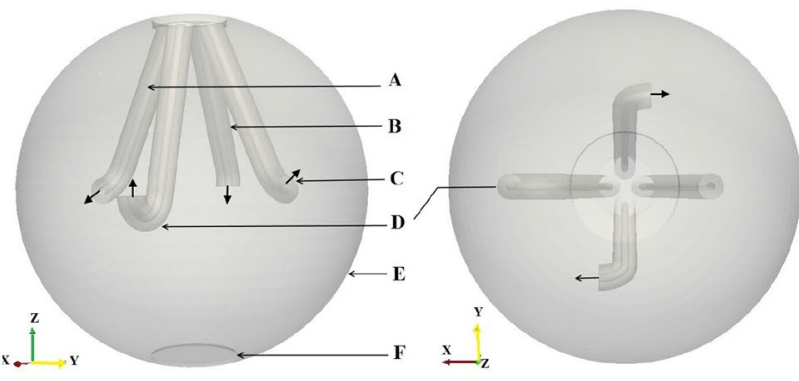

Fig. 1 Geometry of one of the simulated JSR. Nozzles C and A pointing in horizontal direction, length of $21.4 \mathrm{~mm}$, the orientation of the tube leading to the orifice from the $x-z$ plane of $23^{\circ}$; Nozzle $\mathrm{D}$ pointing upwards, length of $27.1 \mathrm{~mm}$, the orientation of the tube

mesh was unstructured and refined close to the walls, the inlets, and the outlet where higher flow gradients were expected.

The simulated conditions were oriented on the experimental setup of Ayass et al. [8]. A constant temperature of $298 \mathrm{~K}$ is applied for the inlet boundary and the initial condition, while a zero-gradient condition for the temperature and the species mass fraction is considered at the walls. For the pressure, an atmospheric condition was considered at the outlet and zero-gradient at the walls. Regarding the velocity field, we applied zero initial conditions together with no-slip boundary conditions at the walls, a constant value with a top-hat profile at the entrance of nozzles' orifices and zero-gradient at the outlet. The Reynolds number (for $\mathrm{CO}_{2}$ having kinematic viscosity of $10.3 E-6 \mathrm{~m}^{2} / \mathrm{s}$ ) based on the averaged inlet velocity of $26.9 \mathrm{~m} / \mathrm{s}$ and the orifice diameter of $1 \mathrm{~mm}$ is calculated as 2620 and the reactor diameter of $40 \mathrm{~mm}$ as 10400 . Furthermore, the time-averaged Mach number reaches 0.18 at the nozzles' orifice where the gas velocity is the highest.

In accordance with the experiment, a constant flow of $\mathrm{CO}_{2}$ is first injected inside the spherical chamber by aid of four inclined and crossed nozzles situated in the equatorial plane. Then, a solenoid valve sharply cuts off the $\mathrm{CO}_{2}$ stream and injects suddenly a continuous flow of $\mathrm{N}_{2}$ inside the chamber with different constant volumetric flows ranging from $18 \mathrm{~cm}^{3} / \mathrm{s}$ to $180 \mathrm{~cm}^{3} / \mathrm{s}$. In accordance with the experiment by Ayass et al. [8], a constant flow of $\mathrm{CO} 2$ is first injected inside the spherical chamber by aid of four inclined and crossed nozzles located in the equatorial plane. The outlet concentration of the tracer is then calculated as a function of time. In our previous research [7], the results of of the numerical calculations of the RDT were validated versus experimental data from literature [8] for different flow rates and ambient conditions. In this numerical study, we have kept the value of mean residence time constant ( $\tau=0.37 \mathrm{~s}$ ) in order to understand the effective parameters which improve the mixing quality inside the
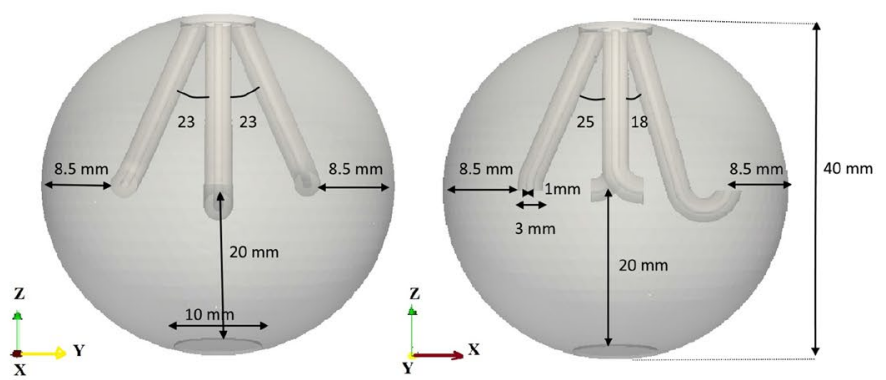

leading to the orifice from the $y-z$ plane of $18^{\circ}$; Nozzle B pointing downwards, length of $22.8 \mathrm{~mm}$, the orientation of the tube leading to the orifice from the $y-z$ plane of $25^{\circ}$; Spherical chamber $\mathrm{E}$, diameter of $40 \mathrm{~mm}$; Outlet $\mathrm{F}$, diameter of $10 \mathrm{~mm}$

JSR. That means the volumetric flow rate and the reactor volume were both considered constant in terms of varied thermodynamic conditions (i.e. Sect. 3.2). However, these variables were changed in terms of varied geometrical parameters (i.e. Sect. 3.2).

Aiming to find an optimized JSR design, we performed a parametric study considering geometrical and thermophysical parameters. More specifically, we investigated the effects of varying the reactor diameter and the nozzle diameter together with an increased operation pressure and various temperatures on the mixing. Table 1 presents a list of conditions considered in the parametric study.

\subsection{Grid resolution}

Figure 2 shows an overview of the unstructured grid over the 3D computational domain. A combination of hexahedral, polyhedral, and prism-shaped cells were generated non-uniformly by snappyHexMesh [23]. Since the impinging jets provide significant flow gradients the grid is refined in the vicinity of nozzles orifices and the chamber walls and also in the region of the outlet. In total, the mesh contains approximately one million cells each of a volume between $3.8 \times 10^{-15} \mathrm{~m}^{3}$ and $3.5 \times 10^{-9} \mathrm{~m}^{3}$, see Fig. $2 \mathrm{c}$.

Table 1 Operating conditions in terms of the diameter of the spherical chamber $(D)$, the diameter of the nozzles $(d)$, temperature $(T)$, pressure $(P)$, and volumetric flow rate $(Q)$

\begin{tabular}{llllc}
\hline$D(\mathrm{~mm})$ & $d(\mathrm{~mm})$ & $T(\mathrm{~K})$ & $P(\mathrm{bar})$ & $Q\left(\mathrm{~cm}^{3} / \mathrm{s}\right.$ \\
\hline 40 & 1 & 298 & 1 & 84.6 \\
60 & 1 & 298 & 1 & 285.5 \\
35 & 1 & 298 & 1 & 56.6 \\
40 & 1.5 & 298 & 1 & 37.6 \\
40 & 1 & 400 & 1 & 84.6 \\
40 & 1 & 700 & 1 & 84.6 \\
40 & 1 & 298 & 10 & 84.6 \\
\hline
\end{tabular}




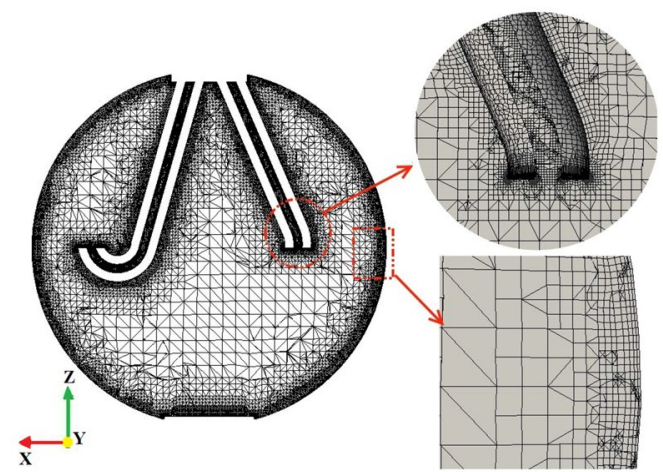

(a)

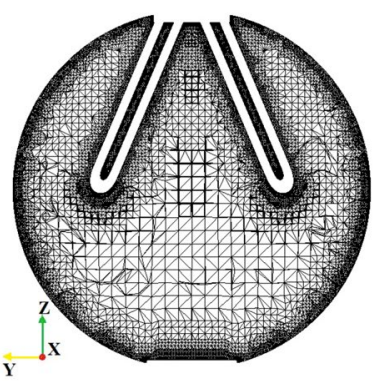

(b)

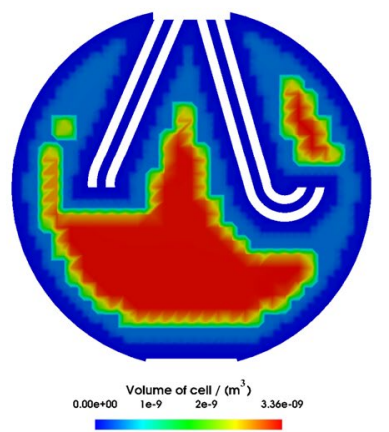

(c)
Fig. 2 Slices of the computational grid in (a) the $x-z$ and (b) the $y-z$ plane. More cells are distributed in the vicinity of the nozzles and chamber walls. The averaged volume of the cells in the $x-z$ crosssection is depicted in (c)

In order to determine whether the wall-adjacent cells are close enough to the reactors solid boundaries we refer to the non-dimensional quantity $y^{+}=y u^{*} / v$. In this equation, $y$ and $v$ are the distance of the center of the closest mesh cell from the wall and the fluid kinematic viscosity, receptively. Furthermore, $u^{*}$ represents the friction velocity at the wall which is defined as a function of wall shear stress, $\tau_{p}$, and density as $u^{*}=\left(\tau_{p} / \rho\right)^{1 / 2}$. In this study, the closest grid point to the nozzles' walls are located at $y^{+}=0.005-0.032$ and to the wall of the spherical part of the chamber at $y^{+}=0.002$. Due to the grid refinement close to the walls generated by "SnappyHexMesh" tools, the distance $y^{+}$is smaller than 1. Therefore, the grids and filter are enough fine to resolve the large-scale motions in the near-wall regions [24].

Additionally, the characteristic size of the Smagorinsky model filter which is defined as a cube root of the minimum cell volume, $0.015 \mathrm{~mm}$, shows that the viscous sublayers of the wall-bounded flows were well resolved on the numerical grid [25].

Further, a commonly used quantity in the characterization of turbulence are the Taylor microscale eddies which are intermediate in size between the smallest eddies
Table 2 Calculation of discretization error of the time-averaged velocity, $V_{\text {ave, }}$ and the mean residence time, $\tau$

\begin{tabular}{lll}
\hline & $V_{\text {ave }}$ & $\tau$ \\
\hline$h_{1}$ & $2.9 \times 10^{-11} \mathrm{~m}$ & $2.9 \times 10^{-11} \mathrm{~m}$ \\
$h_{2}$ & $6.61 \times 10^{-11} \mathrm{~m}$ & $6.61 \times 10^{-11} \mathrm{~m}$ \\
$h_{3}$ & $1.28 \times 10^{-10} \mathrm{~m}$ & $1.28 \times 10^{-10} \mathrm{~m}$ \\
$r_{21}$ & 2.72 & 2.72 \\
$r_{32}$ & 1.93 & 1.93 \\
$g_{1}$ & $1.125 \mathrm{~m} / \mathrm{s}$ & $0.339 \mathrm{~s}$ \\
$g_{2}$ & $0.982 \mathrm{~m} / \mathrm{s}$ & $0.369 \mathrm{~s}$ \\
$g_{3}$ & $0.956 \mathrm{~m} / \mathrm{s}$ & $0.374 \mathrm{~s}$ \\
$P O$ & 1.07 & 1.14 \\
$e_{\mathrm{a}}$ & $12 \%$ & $8.8 \%$ \\
$G C I_{\text {fine }}$ & $7 \%$ & $5.1 \%$ \\
\hline
\end{tabular}

of Kolmogorov scale and largest eddies of the integral scale [25]. The Taylor microscale, $I_{\lambda}$, is computed as $I_{\lambda} / I_{0}=\sqrt{10} R e_{T}^{-1 / 2}$ where the turbulence Reynolds number, $R e_{T}$, is calculated as $u_{0} I_{0} v^{-1}$. Here, $I_{0}$ and $v$ correspond to the reactor diameter (because of the generation of the largest eddies) and kinematic gas viscosity, respectively. The turbulence intensity $u_{0}$ is approximated by $5 \%$ of the mean velocity at the nozzle's orifice [26]. Therefore, the length scale of the Taylor eddies can be estimated to $2.1 \mathrm{~mm}$. This value is 3.36 times larger than the average cell size used in the computations which indicates an appropriate grid resolution.

In order to control the numerical uncertainty and to estimate the accuracy of the discretization, a grid-refinement study on progressively reduced mesh size is performed. According to Roache [27], we first select three significantly different sets of grids and then run simulations to determine the values of key variables important to the objective of the simulation study. It is desired that the grid resolution increment leads to the convergence of the solution.

As reported in Table 2, the solution variable $g_{i}$ which represents the magnitude of the average velocity and mean residence time has been calculated using LES for three grids consisting of $1 \mathrm{M}, 490 \mathrm{k}$, and $240 \mathrm{k}$ cells, respectively. The mean residence time is obtained for the simulated reactor defined through Eq. 6. The apparent order $P O$ can be directly extracted from the three solutions $g_{1}, g_{2}$, and $g_{3}$ on the three meshes of an average cell size of $h_{1}, h_{2}$, and $h_{3}$ with a constant grid refinement ratio of $r_{32}=h_{3} / h_{2}$ and $r_{21}=h_{2} / h_{1}$ using Eqs. $(6,7,8)$.

$P O=\frac{\left|\ln \left(\left|\frac{g_{3}-g_{2}}{g_{2}-g_{1}}\right|+W(P O)\right)\right|}{\ln \left(r_{21}\right)}$, 
$W(P O)=\ln \left(\frac{r_{21}^{P O}-s}{r_{32}^{P O}-s}\right)$

$s=1 \cdot \operatorname{sgn}\left(\frac{g 3-g 2}{g 2-g 1}\right)$

Equation (6) is solved using fixed-point iteration, with the initial guess equal to the first term [28]. Further, an estimation of the error $e_{\mathrm{a}}$ between the fine grid solution and the unknown exact solution can be approximated by

$e_{\mathrm{a}}=\left|\frac{g_{1}-g_{2}}{g_{1}}\right|$.

From this, the numerical uncertainty on the finest grid can be evaluated by the Grid Convergence Method (GCl). The fine-grid convergence index, $\mathrm{GCl}_{\text {fine }}$ [28] is estimated as follows,

$G C l_{\text {fine }}=\frac{1.25 e_{\mathrm{a}}}{r_{21}^{P O}-1}$.

According to Table 2, the apparent order of discretization for the average velocity is 1.07 and for the mean residence time it is 1.71 which are in the expected range [28]. Since the grid acts as an implicit filter in this study, the $\mathrm{GCI}$ represents both the numerical and modeling errors. Even though we cannot separate the contribution of the errors, their sum has a minor effect on our key results [29].

Another method for discretization error estimation is to illustrate the numerical error $e$ (subtracting the solution on the fine and the course mesh) regarding calculation of averaged $\mathrm{CO}_{2}$ mass fraction versus total number of cells $\mathrm{N}$ $(240 \mathrm{k}, 490.4 \mathrm{k}$, and $1.2 \mathrm{M})$ which is shown in Fig. 3. Furthermore, the slope of the line depicted in this figure shows the order of discretization accuracy which is earlier specified as second order for the spatial derivatives.

\section{Numerical results}

In this section, we first characterize the unsteady flow field and the species distribution inside the spherical chamber of the JSR. Since we have assumed a constant temperature on the boundaries, a significant temperature inhomogeneity is not expected. Then we present the results of the effects of the reactor geometry together with the elevated pressure and temperature on mixing enhancement.

\subsection{Flow characteristic inside the JSR}

In our previous LES [7], a top-hat profile, i.e., a constant uniform velocity of $26 \mathrm{~m} / \mathrm{s}$ for the incoming gas, was

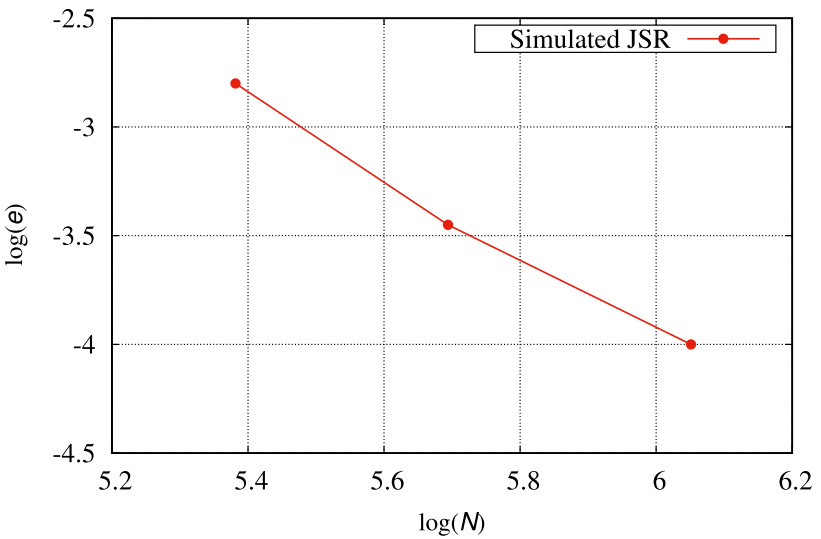

Fig. 3 Grid convergency test for the operating condition of first row of Table 1. The numerical error represents the difference of the solution to the finest mesh solution in terms of the average $\mathrm{CO}_{2}$ mass fraction

assumed at the outlets of the jets. In the present simulations we also model the in-nozzle flow in order to capture the flow field in the reactor more realistically. Thus, a tophat profile is assumed at the beginning of each nozzle. Then, in downstream direction turbulent velocity profile is developed inside the nozzle. The top-hat and the developed velocity profiles are illustrated in Fig. $4 a$ and $b$, respectively.

The time-averaged streamlines extracted from the instantaneous velocity field are visualized in Fig. 5 indicating the direction of the flow. The averages in this study are derived from instantaneous data that correspond to time instances where the flow is already statistically stationary but the injection of $\mathrm{N}_{2}$ has not started yet. In this case (which corresponds to the operating condition given in the first row of Table 1), the averaging time scale of $30 \mathrm{~ms}$ is much larger than the time scale based on physical boundaries of the flow of $0.8 \mathrm{~ms}$ (e.g. diameter of the reactor divided by the highest velocity inside the chamber).

According to Fig. 5a and b, the flow impinges to the in-front wall and bends in the counter clockwise direction due to the location of the entry of the nozzles pointing upwards or downwards. Similarly, a circumferential stream is generated by the two other nozzles in $x-z$ plane as it can be inferred from Fig. $5 \mathrm{c}$. Therefore, two circulating flow pattern formed by the orientation of the nozzles aims to efficiently support the mixing within the reactor. Further, the distribution of streamlines using the surface-LIC (Line Integral Convolution) technique is illustrated in appendix A [30, 31].

In order to identify the vortical structures of the flow field, the $Q$-criterion is used which is derived based on the second invariant of velocity gradient tensor $(Q=$ $\left.\left|\Omega^{2}\right|-|S|^{2}\right)$. Here, $S$ and $\Omega$ refer to strain rate tensor and 


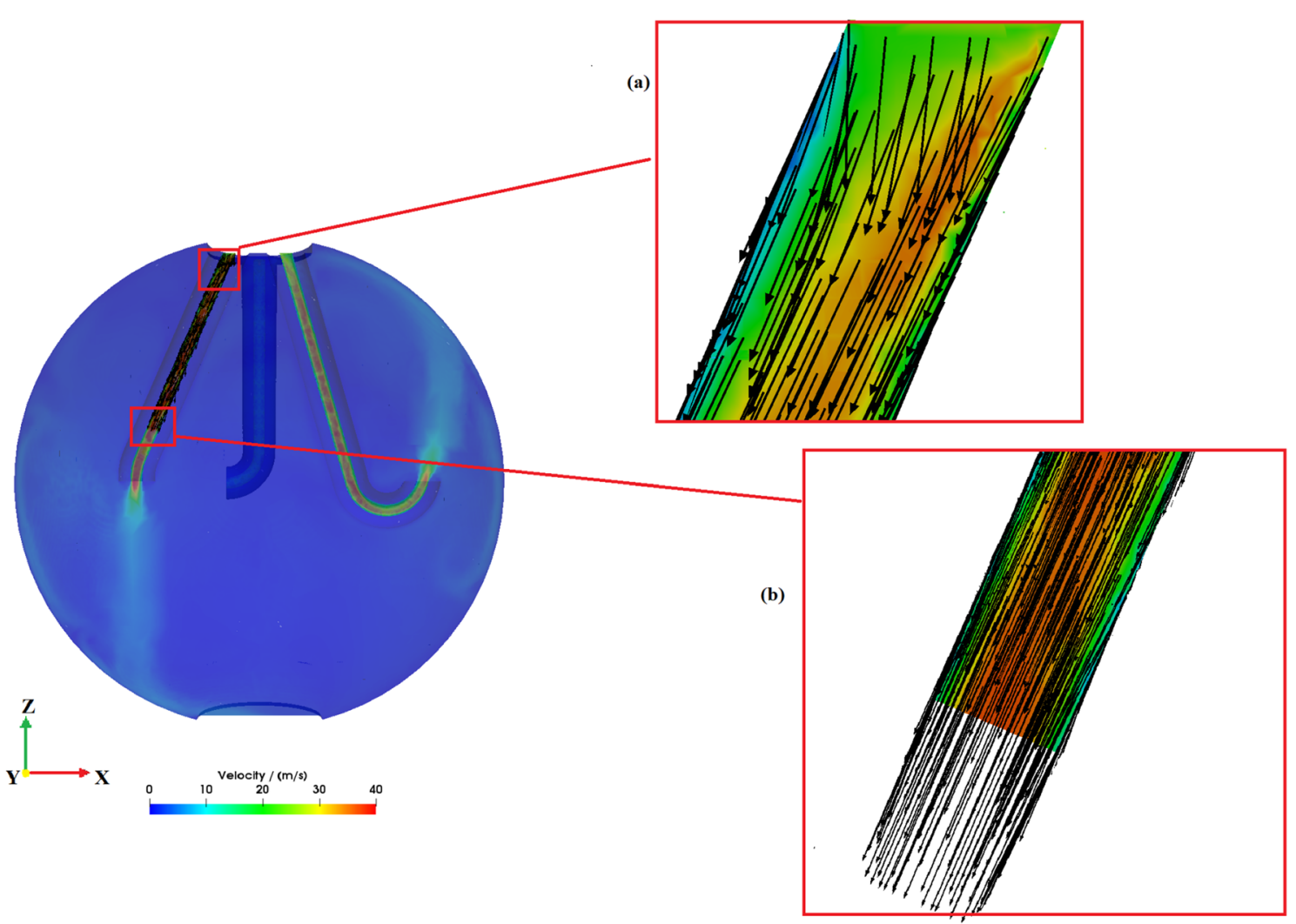

Fig. 4 Velocity field inside the downward nozzle; $\mathbf{a}$ top-hat profile at the entrance of nozzle and $\mathbf{b}$ a developed profile at the cross section close to the orifice

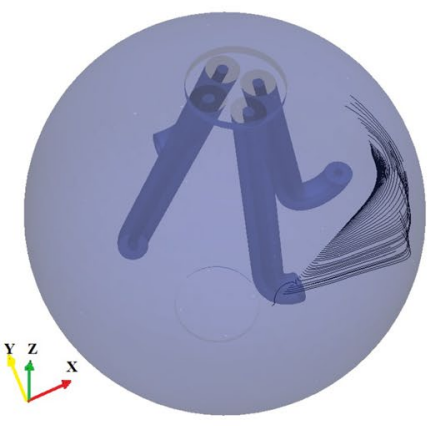

(a)

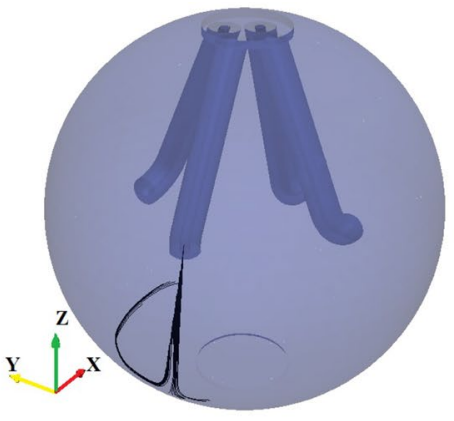

(b)

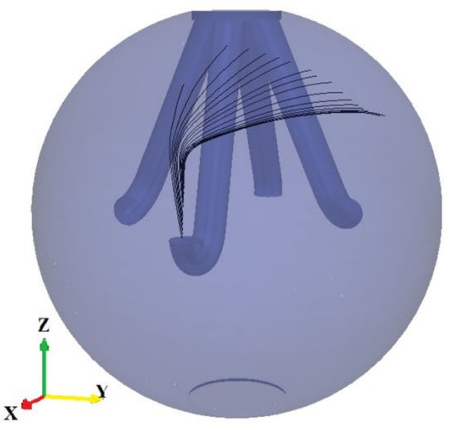

(c)

Fig. 5 Time-averaged streamlines originating from the horizontal nozzle (a) and the downwards and upwards pointing nozzles (b and $\mathbf{c}$ )

rotation tensor, respectively [32]. The positive value of $Q$ represents the existence of vortex inside the chamber since the rotation rate is greater than the strain rate. The distributions of instantaneous $Q$-criterion is presented in the cross section of the $x-z$ plane in Fig. 6 . As expected, a high vorticity is generated at the walls of the nozzles due to the high velocity gradient caused by the assumption of no-slip boundary conditions. Subsequently, the inertial flow at the exit of the nozzles create a vortical structure. As soon as the flow stream starts to bend, it grows in a roller structure and then breaks down into small-scale motions along the wall until it almost decays. In fact, the change of the direction results in the formation of a large circulation bubble downstream of the location of the impingement of the jet to the inner surface of the vessel as discussed previously by Hodzic et al. [33]. It is to be noted that the 


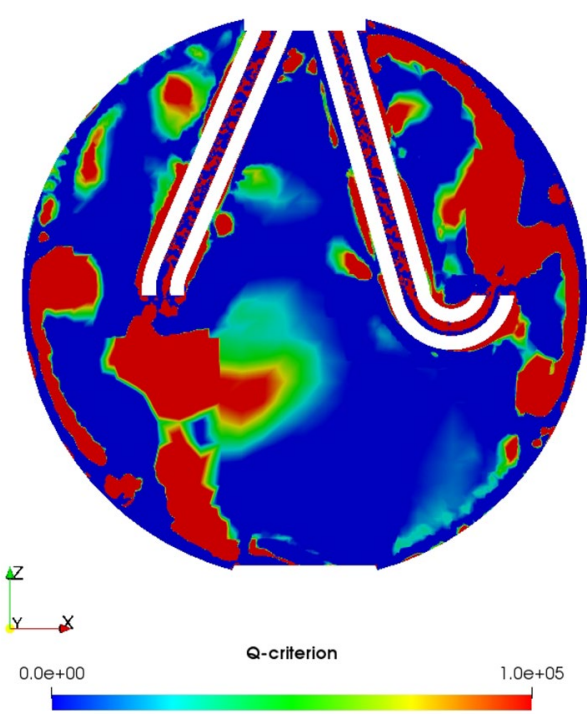

Fig. 6 Visualization of the Q-criterion distribution in the cross-sections described by the $x-z$ plane

LES model helps to predict the eddy structures with different length scales which is not captured by previous RANS simulations [34].

According to Fig. 6, a lower vorticity region is formed in the center of the chamber because of the interaction of the two circumferential flows growing towards the spherical walls and also being away from the walls. As a consequence, a dead zone is created in this location which inhibits a perfectly mixed composition.

The total specific turbulent kinetic energy $k_{\text {tot }}$ consists of the specific turbulent kinetic energy in both the resolved scales $k_{R}$, and the sub-grid scales $k_{\text {sgs }}$, which is given by [35]

$k_{\text {tot }}=k_{R}+k_{\text {sgs }}$.

The specific turbulent kinetic energy of SGS scales can be calculated from the contribution of the SGS turbulence model (Smagorinsky) as follows,

$k_{s g s}=\frac{v_{s g s}^{2}}{\left(C_{k} \Delta\right)^{2}}$,

where $C_{k}$ is the static model coefficient. It is suggested that the LES using eddy viscosity SGS models is a good measure for estimating the grid resolution [36]. Further, the resolved specific turbulent kinetic energy can be evaluated as

$k_{R}=\frac{1}{2} \sum_{j=1}^{3}\left(u_{j}^{\prime}\right)^{2}$.
In the above equation $u_{j}^{\prime}$ denotes the Root Mean Square (RMS) value of the filtered velocity components in the $x, y$, and $z$ directions [25].

According to Pope [25], the resolved turbulent kinetic energy contains $80 \%$ of the total turbulent kinetic energy in a well-resolved LES simulation [37]. Therefore, we investigate the distribution of the resolved specific turbulent kinetic energy in Fig. 7a. As expected the highest turbulence fluctuations which enhance the mixing inside the JSR are present where the jets impinge on the inner surface of the vessel as discussed previously by Hodzic et al. [33]. This behavior indicates the necessity of impinging jets for the mixing inside a JSR. We specified the value of resolved specific turbulent kinetic energy over three points in Fig. 7a for further comparison of cases mentioned in Table 1.

In order to determine how the specific turbulent kinetic energy is distributed over eddies of many sizes (wave numbers) or over many frequencies eddies, we plot the energy spectrum for one point located at the center of the chamber with the coordinate of $[x: 0.0054 \mathrm{~mm}, y: 0 \mathrm{~mm}, z: 0 \mathrm{~mm}]$ in Fig. 7b. According to Sisemore et al. [38], the dissipated energy spectra can be derived as a function of a smoothed Fourier amplitude spectrum. Therefore, in order to plot the energy spectrum, the Fast Fourier Transform (FFT) of the velocity field is calculated over a time domain [0.01s-0.06s] which is larger than the integral time scale [39]. As shown in Fig.7b, the specific kinetic energy density shows a maximum value for the frequency numbers $f$ corresponding to the large energy-containing eddies. Further, the spectra of the velocity follows the $-5 / 3$ law for a wide range of frequencies which indicates a state of fully-developed turbulence. It should be noted that the energy spectrum is shown for frequencies smaller than $10^{5} \mathrm{~s}^{-1}$. That means for those frequencies which are properly resolved by the numerical time step. The Strouhal number $\left(S t=f \cdot D / U_{0}\right)$ varies in the range of [4.9-790] where $D$ is the reactor diameter and $U_{0}$ is the averaged value of the velocity at the nozzles.

\subsection{Parametric investigation of the thermodynamic conditions}

In order to study the influence of thermodynamic conditions on mixing inside the JSR, we elevated the initial and boundary conditions of the temperature to $400 \mathrm{~K}$ and $700 \mathrm{~K}$ at atmospheric pressure, and also the pressure to 10bar at atmospheric temperature, respectively. Since we kept the theoretical mean residence time constant, the inflow velocity is also kept constant at the entrance of nozzles' orifices. To quantify the mixture homogeneity in the JSR, 


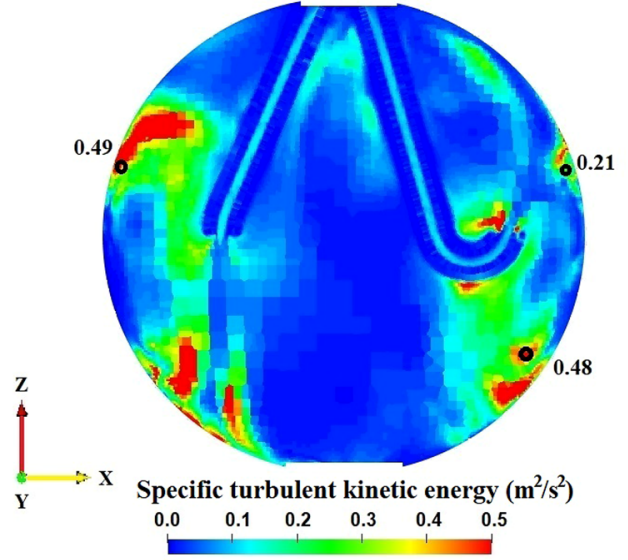

(a)

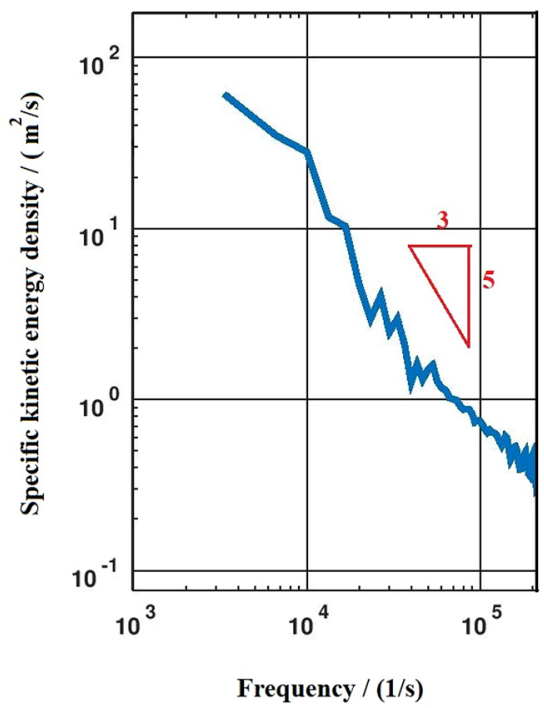

(b)

Fig. 7 a Distribution of the resolved specific turbulent kinetic energy in the $x-z$ plane, $\mathbf{b}$ Specific kinetic energy spectrum at the point located in the center of reactor

we calculated the normalized standard deviation of the $\mathrm{CO}_{2}$ mixture fraction field, $\sigma$, by

$\left.\sigma=\sqrt{\{} \frac{\sum_{i=1}^{\text {Cells }}\left(Y_{i, \mathrm{CO}_{2}}-Y_{\text {mean }, \mathrm{CO}_{2}}\right) \cdot V_{i}}{\sum_{i=1}^{\mathrm{Cells}} Y_{\text {mean }, \mathrm{CO}_{2}} \cdot V_{i}}\right\}$

where $Y_{i}$ is the $\mathrm{CO}_{2}$ mass fraction in cell $i$ and $Y_{\text {mean }}$ is its mean value. The total number of cells is $N$ and $V_{i}$ is the volume of each cell. The normalized standard deviation represents the deviation of the tracer mass fraction from its mean value divided by the mean value in the complete reactor chamber Fig. 8 shows the normalized standard deviation of $\mathrm{CO}_{2}$ mass fraction for the cases of different temperatures. In general, the normalized standard deviation of all cases rises in the beginning until reaching a maximum value and then decreases thereafter. This trends relates to the experimental procedure where $\mathrm{N}_{2}$ is suddenly injected in a JSR chamber being filled with $\mathrm{CO}_{2}$.

Also, in the beginning $(t<0.05 \mathrm{~s})$ a high deviation between the $\mathrm{CO}_{2}$ concentration of the different cases is observed. This deviation becomes less $(t>0.05 \mathrm{~s})$ as fluid leaves the outlet. According to Fig. 8, higher temperature effectuates a higher normalized standard deviation compared to the lower temperature cases. This trend can be explained by the calculated Reynolds number. In this study, molecular viscosity is dependent on temperature through Sutherland's law. Therefore, increasing the temperature causes an increase of the dynamic gas viscosity [40]; because an increase in temperature elevates rapid motion of gas molecules and the collision between them

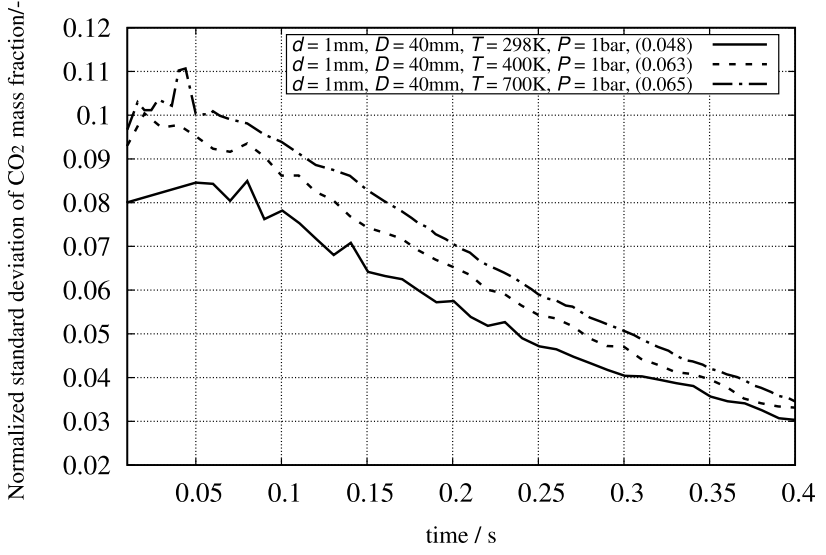

Fig. 8 Temporal evolution of the normalized standard deviation of the tracer mass fraction for different temperatures

and consequently enhances the gas viscosity. Additionally, increasing temperature leads to decrease density of the gas mixture $\left(\mathrm{CO}_{2}-\mathrm{N}_{2}\right)$. As a result, the decreased Reynolds number of the gas mixture inside the chamber tends to damp turbulence and consequently decrease the efficient mixing and the homogeneity of the composition inside the JSR. This fact can be better understood by comparing the distribution of the resolved turbulent kinetic energy in Fig. $7 \mathrm{a}$ and 9 (see the values of the specific turbulent kinetic energy of resolved motions over three identical points for both cases). The total and local specific turbulent kinetic energy at a temperature of $298 \mathrm{~K}$ have greater values compared to the $700 \mathrm{~K}$ case. 


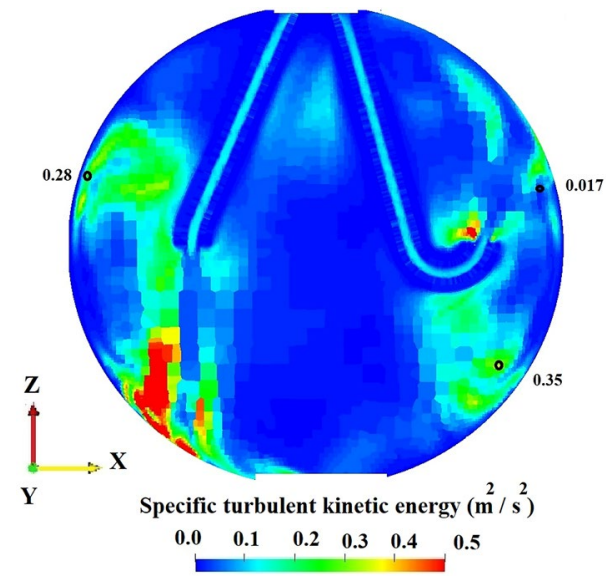

Fig. 9 Distribution of the resolved specific turbulent kinetic energy of the flow field in the $x-z$ plane for the case of $T=700 \mathrm{~K}$

Figure 10 shows that a pressure of 10 bar results in a lower normalized standard deviation of the $\mathrm{CO}_{2}$ mass fraction compared to the case considering ambient pressure. This means an efficient mixing and a better homogeneity are achieved at higher pressures inside the reactor. According to the ideal gas law, higher pressure increases the gas density inside the chamber. This prevents the short circulation of the fluid elements. That means the fluid elements are convected on a short path from the inlets to the outlet without mixing with the rest of the gas inside the JSR's chamber. In fact, higher pressure creates more vortexes over the reactor which supports mixing. The time-averaged velocity contours captured with the LIC method compares the flow field of different pressures in appendix A.

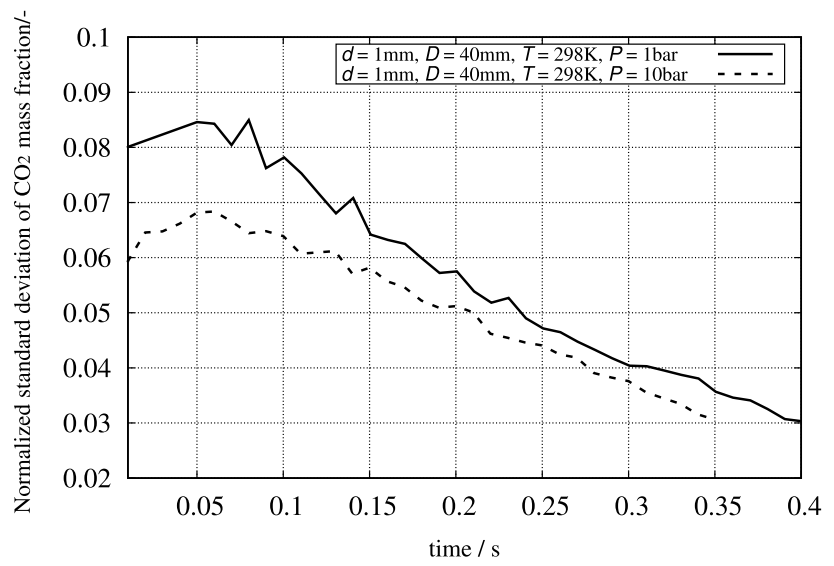

Fig. 10 Temporal evolution of the normalized standard deviation of the tracer mass fraction for an elevated pressure

\subsection{Parametric investigation of the geometrical parameters}

Further, we studied the effects of geometrical parameters on the mixing inside the JSR considering a constant residence time. We changed the diameter of the nozzles to $1.5 \mathrm{~mm}$ and also the diameter of the chamber from $35 \mathrm{~mm}$ to $60 \mathrm{~mm}$ at ambient thermodynamic conditions. Because of the practical limitations, a JSR with a nozzle and chamber diameter of less than $1 \mathrm{~mm}$ and $35 \mathrm{~mm}$ respectively, cannot be built up.

In Fig. 11 the normalized standard deviation of the $\mathrm{CO}_{2}$ mass fraction for different nozzle and reactor diameters is plotted versus time. As can be seen in Fig. 11a, a more homogeneous composition after $t=0.15 \mathrm{~s}$ is achieved by decreasing the chamber diameter. However, the reactor having $D=60 \mathrm{~mm}$ reaches the maximum of standard deviation later (at $t=0.15 \mathrm{~s}$ ) compared to the diameter of $D=40 \mathrm{~mm}$ (at $t=0.05 \mathrm{~s}$ ). Because $\mathrm{N}_{2}$ is injected to a JSR

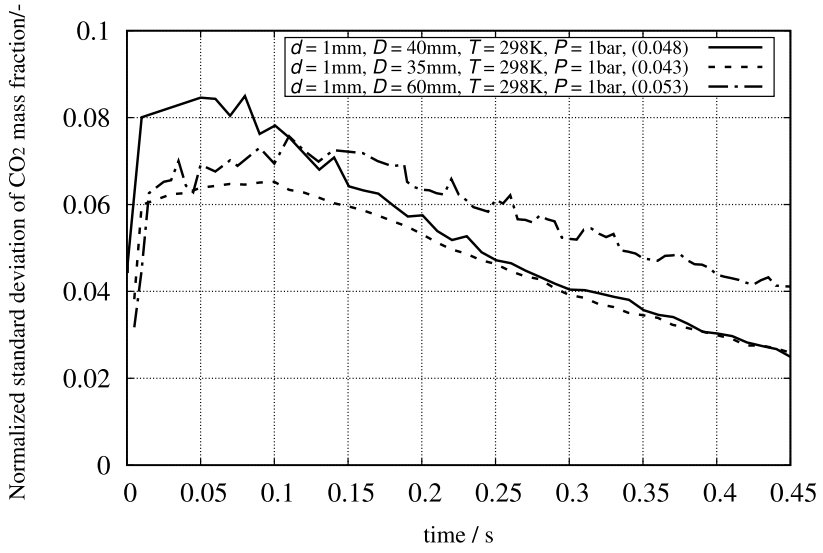

(a)

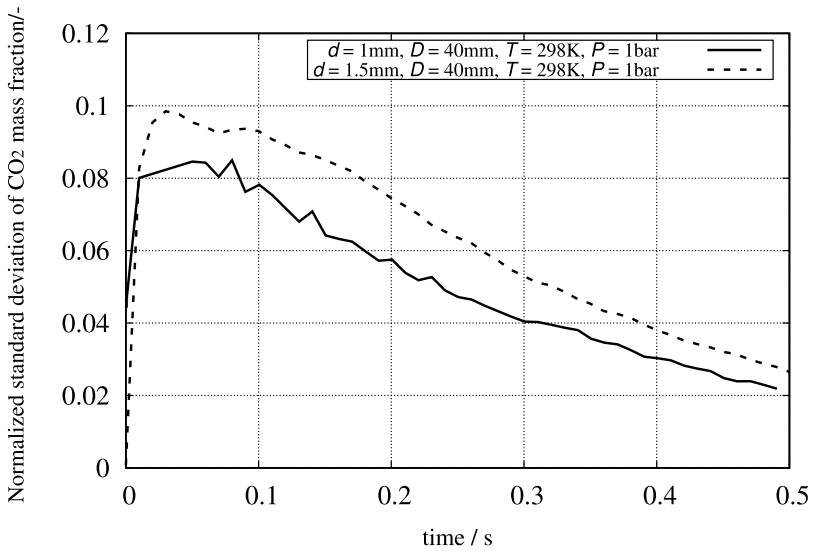

(b)

Fig. 11 Temporal evolution of the normalized standard deviation of the tracer mass fraction for different $\mathbf{a}$ chamber diameters and $\mathbf{b}$ nozzle diameters 
which is already filled with a homogeneous composition of $\mathrm{CO}_{2}$, the fluid needs to travel a longer distance and time to the outlet in the case of larger chamber with the same outlet's diameter of $10 \mathrm{~mm}$ and therefore the maximum value is achieved later. The smaller chamber in the case of $D=35 \mathrm{~mm}$ with the same outlet's diameter of $10 \mathrm{~mm}$ leaves the flow faster (the time lag between the inlets and outlet is shorter than the case of $D=40 \mathrm{~mm}$ ) and does not let to generate an intensive dead zones inside the JSR, therefore it provides a well-stirred mixture.

The normalized $\mathrm{CO}_{2}$ standard deviation of the case of a nozzle diameter of $d=1.5 \mathrm{~mm}$ is compared to $d=1 \mathrm{~mm}$ for a constant chamber diameter of $D=40 \mathrm{~mm}$ in Fig. $11 \mathrm{~b}$. The result shows that the larger diameter of the nozzle cannot improve the mixing inside the JSR. The reason can be understood as follows. In order to keep the theoretical mean residence time constant, the volumetric inflow rate is also kept constant. Therefore, the initial velocity modeled at the entry of the nozzles in the case of $d=1.5 \mathrm{~mm}$ is less than the initial velocity in the case of $d=1 \mathrm{~mm}$. Smaller velocity for the case of larger nozzle diameter of $d=1.5 \mathrm{~mm}$ generates a more intensive dead zone at the center of the JSR as illustrated in Fig. 12. In the dead regions a very small exchange of material happens inside the reactor with insufficient molecular mixing [41].

\section{Proposed improved geometry}

In order to improve the mixing quality, we added two injectors of a diameter of $d=1 \mathrm{~mm}$ to the standard four nozzle design introduced by Dagaut et al. [11]. An overview of this suggested setup having six nozzles is given in Fig 13. One of the advantages of this geometry is that the two nozzles pointing to the center breakdown the

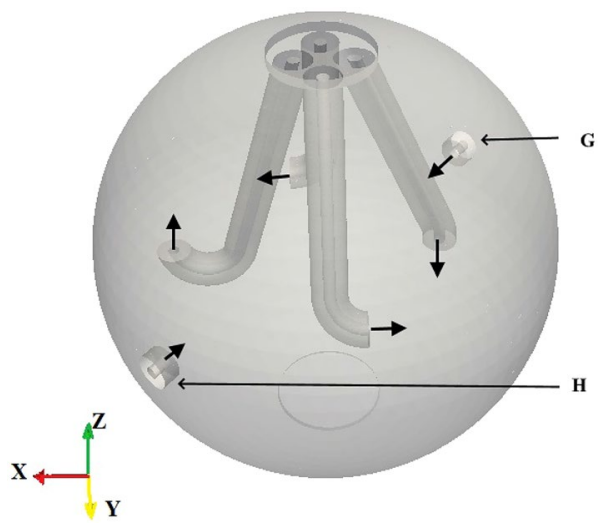

Fig. 13 Proposed new design of the JSR: two additional nozzles (G and $\mathrm{H}$ ) pointing to the center

dead-zones at the center of the JSR which, based on the discussion above, are mainly responsible for the nonhomogeneity. Figures $14 a$ and b compare two cross sections of the time-averaged tracer mass fraction for cases of four and six nozzles in the $x-z$ and $x-y$ planes, respectively. Comparing the values of the averaged tracer mass fraction over three points shows that the amount of non-homogeneity in the case of six nozzles is decreased compared to the case of four nozzles. In fact, the dead-zone is removed from the reactor by applying two more jets pointing to the center.

Comparing the resulting curves for the normalized standard deviation of the $\mathrm{CO}_{2}$ mass fraction in Fig. 15 indicates that a more uniform composition is achieved for the new design of the JSR compared to the typical JSR. The lower normalized standard deviation in the case of six nozzles might be explained by the vortexes at the center of the JSR induced by the high inertia of the jets.
Fig. 12 Dead spaces visible in the time-averaged distribution of the $\mathrm{CO}_{2}$ mass fraction ( [0.08s - 0.09s] after tracer injection) over the $x-y$ plane for the cases of $d=1 \mathrm{~mm} ; D=40 \mathrm{~mm}$ and $d=1.5 \mathrm{~mm} ; D=40 \mathrm{~mm}$

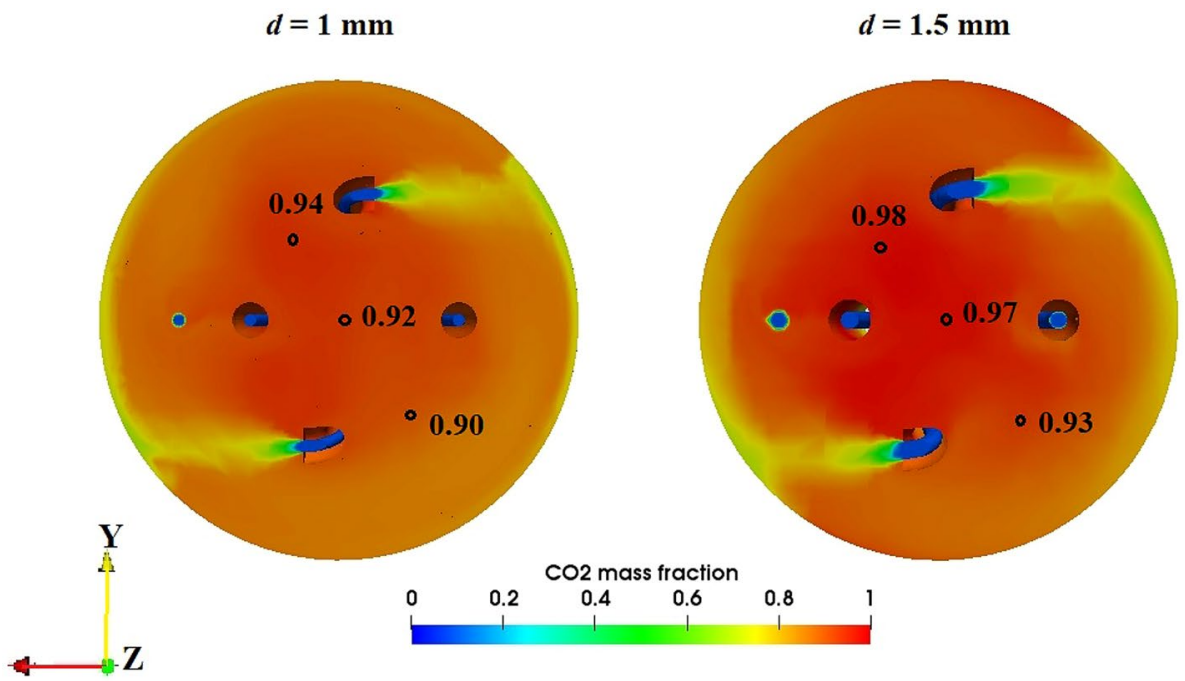

SN Applied Sciences A SPRINGER NATURE journa 
Fig. 14 Time-averaged concentration of $\mathrm{CO}_{2}$ in the cross-sections described by the (a) $x-z$ plane, (b) $x-y$ plane: Left: Six nozzles JSR, Right: Four nozzles JSR

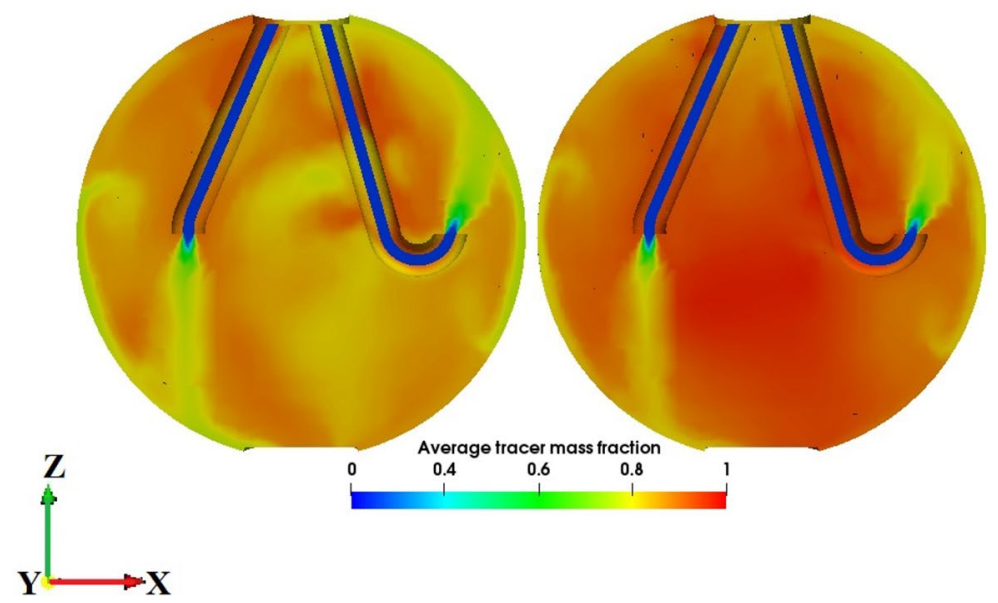

(a)

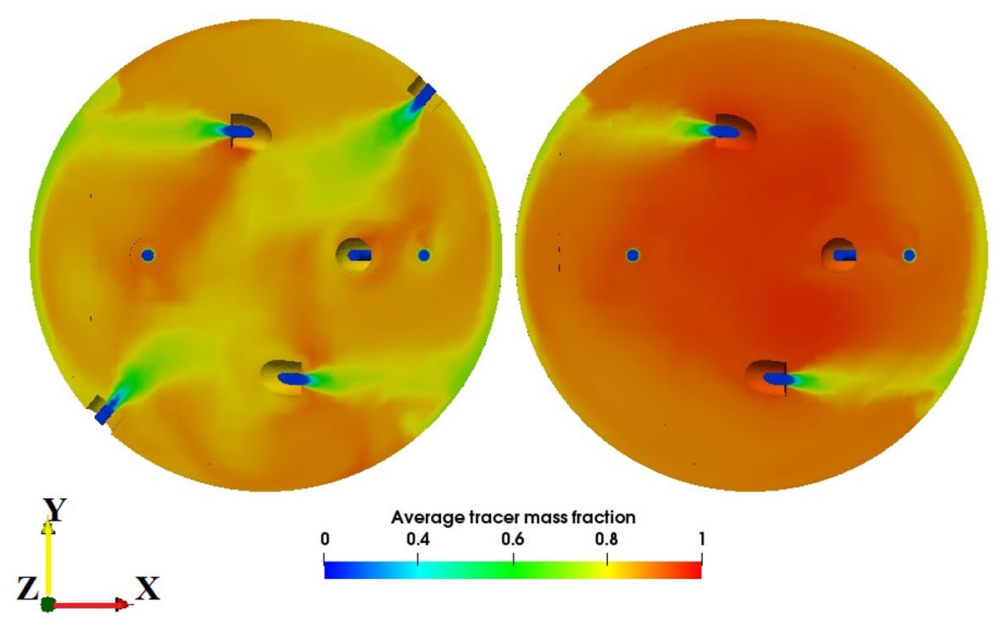

(b)

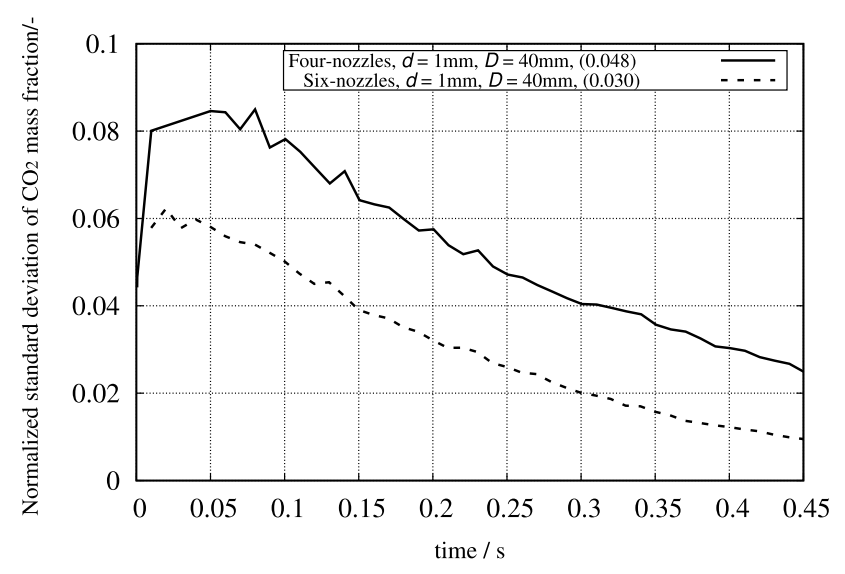

Fig. 15 Temporal evolution of the normalized standard deviation of the tracer mass fraction for the reactors with four and six nozzles
We are aware that the JSR design of Dagaut [11] requires a premixed chamber of the gases before they enter the reactor spherical chamber; however, adding two nozzles will improve the mixing inside the reactor. The time-averaged streamlines using LIC technique and resolved kinetic energy are further discussed in the appendix A.

\section{Conclusions}

The mixing process and spatial homogeneity inside a spherical Jet-Stirred Reactor (JSR) have been studied via well-resolved large eddy simulations. First, the grid resolution study is investigated and then the characteristic of flow field created by four impinging jets have been captured through a numerical method. Analyzing stream-lines and velocity distributions with LIC method introduced the details of the flow field pattern which could 
not be obtained by limited optical accessibility of experiments. Further, studying the time-averaged distribution of the resolved specific turbulent kinetic energy and vorticity shows that the highest turbulence fluctuations and, therefore, best mixing occur where the jets impinge on the inner surface of the vessel. Results of the energy spectrum following $-5 / 3$ law for a wide range of frequencies represents a state of fully developed turbulence. Second, a parametric study in terms of geometrical parameters and thermodynamic conditions has been performed in order to improve mixing inside the JSR. According to the results of the normalized standard deviation of the tracer mass fraction and the resolved specific turbulent kinetic energy, higher temperatures might not follow the ideal reactor. However, increasing the pressure above atmospheric results in a lower value of the normalized standard deviation of $\mathrm{CO}_{2}$ which indicates an improvement to the mixing. Therefore, the four-jets JSR with nozzle diameters of $1 \mathrm{~mm}$ and chamber diameter $40 \mathrm{~mm}$ at atmospheric pressure up to 10 bar create a nearly homogeneous composition.

Finally, we proposed a new JSR design by adding two nozzles pointing to the center of the reactor. Our simulations predicted that this design would breakdown the dead-zones at the center of the JSR and significantly improve the mixing inside the reactor.

Acknowledgements This work was partially supported by the INNO INDIGO programme BiofCFD.

Funding Open Access funding enabled and organized by Projekt DEAL.

\section{Declarations}

Conflict of interest The authors declare that they have no conflict of interest.

Open Access This article is licensed under a Creative Commons Attribution 4.0 International License, which permits use, sharing, adaptation, distribution and reproduction in any medium or format, as long as you give appropriate credit to the original author(s) and the source, provide a link to the Creative Commons licence, and indicate if changes were made. The images or other third party material in this article are included in the article's Creative Commons licence, unless indicated otherwise in a credit line to the material. If material is not included in the article's Creative Commons licence and your intended use is not permitted by statutory regulation or exceeds the permitted use, you will need to obtain permission directly from the copyright holder. To view a copy of this licence, visit http://creativecommons. org/licenses/by/4.0/.

\section{Appendix A: LIC method}

\section{Illustration of streamline using LIC}

Figure 16 displays the time-averaged velocity contour of the rotating confined flow over the time of $[0.1 s-0.4 \mathrm{~s}]$ after the injection of $\mathrm{CO}_{2}$. The streamlines shown in Fig. 16 are extracted using the surface-LIC (Line Integral Convolution) technique which was introduced by Cabral et al. [30] and was subsequently developed by Loring et al. [31]. This technique uses an image vector field algorithm which is gradually extended in several software packages, e.g. paraview [31], to visualize the flow traces efficiently. The obvious lack of vortices in the center of reactor motivates the development of an optimal configuration of multiple pairs of impinging jets.

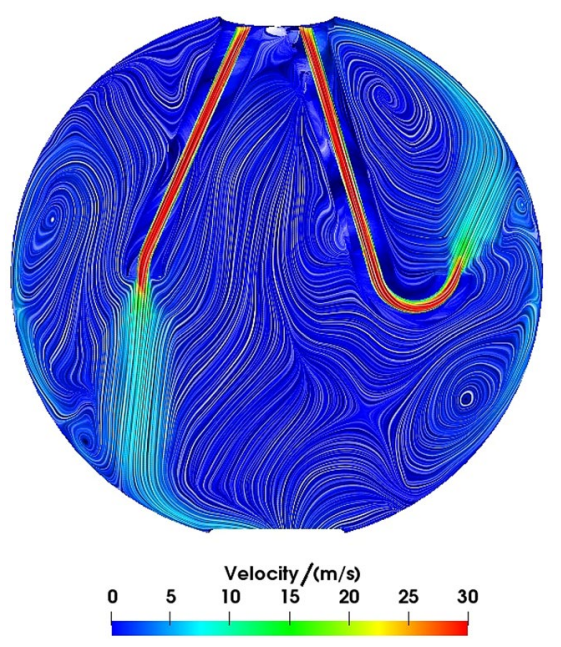

Fig. 16 Streamlines of the time-averaged flow field visualized with the LIC method in the cross-sections described by the $x-z$ axes 


\section{Comparing the LIC contours in different pressures}

The time-averaged velocity contours captured with the LIC method compares the flow structures close to the

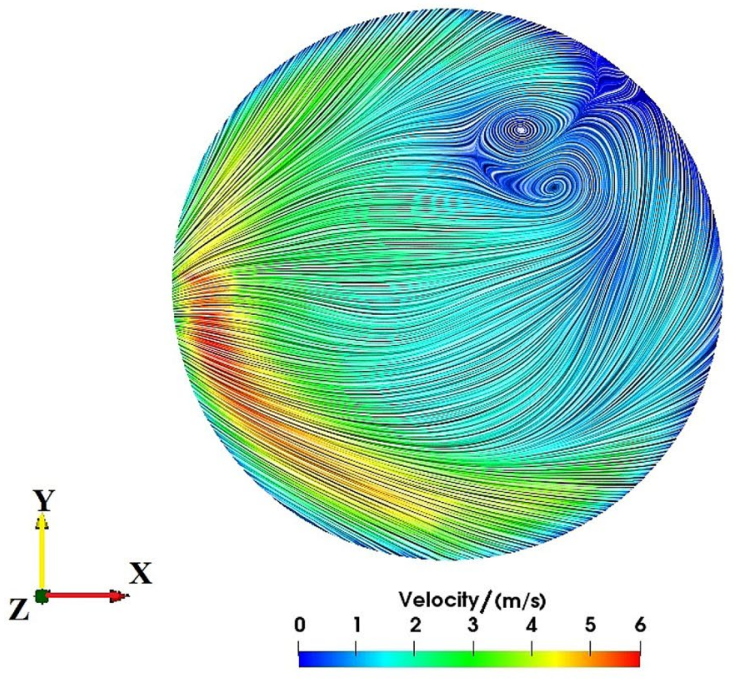

(a) outlet for a pressure of 1 bar in Fig. 17a and pressure of $10 \mathrm{bar}$ in Fig. 17b. According to this figure, three vortexes appeared over the reactor in the case of high pressure which improve the mixing.

Fig. 17 Time-averaged LES computed velocity contour ( [0.08s - 0.09s] after tracer injection) extracted with the LIC method in the cross section described by the $x$ - $y$ axes at the outlet for the case of $p=1$ bar (a) and $p=10$ bar (b)

\section{Distribution of streamline using LIC and the resolved specific turbulent kinetic energy of the suggested setup}

The LIC visualization illustrated in Fig. 18a shows that these vortexes intensify the turbulent structures over the reactor which leads to an efficient mixing. This suspicion is further supported by the resolved specific turbulent kinetic energy field in Fig. 18b that exhibits a high resolved kinetic energy in the center of the reactor.

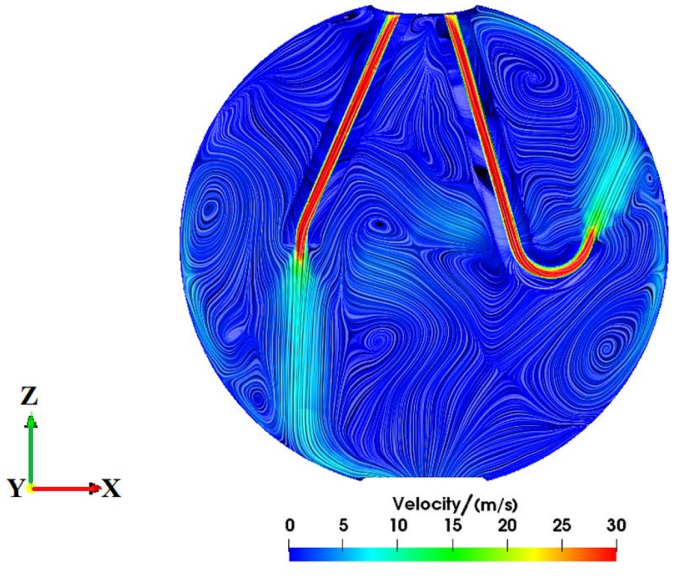

(a)

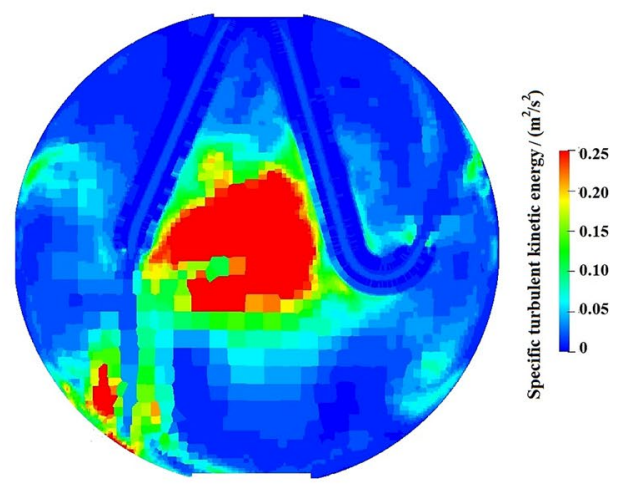

(b)

Fig. 18 Flow field resulting from the new JSR design comprising six nozzles in the cross-section described by the $x$ and $z$ axes. a Time-averaged velocity vectors field visualized with the LIC method. $\mathbf{b}$ Turbulent kinetic energy field 


\section{References}

1. Herbinet O, Dayma G (2013) Jet-stirred reactors Cleaner Combustion. Springer, Berlin

2. Zhang Z, Zhao H, Cao L, Li G, Ju Y (2018) Kinetic effects of n-heptane addition on low and high temperature oxidation of methane in a jet-stirred reactor. Energ. Fuel 32(11), 11970-11978

3. Fogler HS (1999) Elements of Chemical Reaction Engineering. Prentice-Hall International, London

4. Gil I, Mocek P (2012) CFD analysis of mixing intensity in jet stirred reactors. Chem. Process Eng. 33(3), 397-410

5. Davani AA, Ronney PD (2017) A new jet-stirred reactor for chemical kinetics investigations. 10th U.S. National Combustion Meeting, Maryland

6. Zhang T, Zhao H, Ju Y (2018) Numerical studies of novel inwardly off-center shearing jet-stirred reactor. AIAA journal 56(9):3388-3392

7. Esmaeelzade G, Moshammer K, Fernandes R, Markus D, Grosshans $\mathrm{H}$ (2019) Numerical study of the mixing inside a jet stirred reactor using large eddy simulations. Flow Turbul. Combust. 102(2), 331-343

8. Ayass WW, Nasir EF, Farooq A, Sarathy SM (2016) Mixing-structure relationship in jet-stirred reactors. Chem. Eng. Res. Des. 111:461-464

9. Oliveria D, De Toni AR, Cancino LR, Oliveira AA, Oliveira E, I., R. M. Computational fluid dynamics analysis of different geometries for a jet stirred reactor for fuel research. Proceedings of COBEM

10. MacMullin RB, Weber M (1935) The theory of short circulating in continuous flow mixing vessels in series and the kinetics of chemical reactions in such systems. Trans. Am. Inst. Chem. Eng. 31:409-458

11. Dagaut $P$, Cathonnet $M$, Rouan JP, Foulatier R, Quilgars $A$, Boettner JC, Gaillard F, James H (1986) A jet-stirred reactor for kinetic studies of homogeneous gas-phase reactions at pressures up to ten atmospheres ( $\approx 1 \mathrm{mpa})$. J. Phys. E: Sci. Instrum. 19(3):207

12. Ghorbani A, Steinhilber G, Markus D, Maas U (2015) Ignition by transient hot turbulent jets: An investigation of ignition mechanisms by means of a PDF/REDIM method. Proc. Combust. Inst. 35(2), 2191-2198

13. OpenFOAM (2013) http://www.openfoam.com/

14. De Almeida YP, da Cunha Lage PL, Silva LF LR (2013) Validation of LES simulation of a turbulent radiant flame using OpenFOAM. In Proceedings of COBEM

15. Issa RI (1986) Solution of the implicitly discretised fluid flow equations by operator-splitting. J. Comput. Phys. 62(1), 40-65

16. Patankar SV (1980) Numerical Heat Transfer and Fluid Flow. Hemisphere, New York

17. Burcat A, Ruscic B, et al. (2005) Third millenium ideal gas and condensed phase thermochemical database for combustion with update from active thermochemical tables. Tech. rep., Argonne National Lab., United States

18. Prokein D, von Wolfersdorf J (2019) Numerical simulation of turbulent boundary layers with foreign gas transpiration using OpenFOAM. Acta Astronautica 158:253-263

19. Darwish M, Orazi L, Angeli D (2019) Simulation and analysis of the jet flow patterns from supersonic nozzles of laser cutting using OpenFOAM. Int. J. Adv. Manuf. Tech. 102(9), 3229-3242

20. Smagorinsky J (1963) General circulation experiments with the primitive equations: I the basic equations. Mon Weather Rev., 91(3), pp. 99-164
21. Lysenko DA, Ertesvåg IS, Rian KE (2012) Large-eddy simulation of the flow over a circular cylinder at Reynolds number 3900 using the OpenFOAM toolbox. Flow Turbul. Combust. 89(4), 491-518

22. Wolf $D$, Resnick W (1963) Residence time distribution in real systems. Ind. Eng. Chem. Fundamen. 3(4), 287-293

23. Gisen D (2014) Generation of a 3D mesh using snappy hex mesh featuring anisotropic refinement and near-wall layers. ICHE, Hamburg, Germany

24. Ceresiat L, Grosshans H, Papalexandris MV (2019) Powder electrification during pneumatic transport: The role of the particle properties and flow rates. J. Loss Prev. Process Ind. 58:60-69

25. Pope SB (2000) Turbulent Flows. Cambridge University Press

26. Grosshans H, Cao L, Fuchs L, Szász R-Z (2017) Computational sensitivity study of spray dispersion and mixing on the fuel properties in a gas turbine combustor. Fluid Dyn. Res. 49(2):025506

27. Roache PJ (1997) Quantification of uncertainty in computational fluid dynamics. Annu. Rev. Fluid Mech. 29(1), 123-160

28. Celik IB, Ghia U, Roache PJ, Freitas CJ, Coleman H, Raad PE (2008) Procedure for estimation and reporting of uncertainty due to discretization in CFD applications. J. Fluid Eng-T ASME 130(7), 78001-78004

29. Bose ST, Moin P, You D (2010) Grid-independent large-eddy simulation using explicit filtering. Phys. Fluids 22(10):105103

30. Cabral B, Leedom LC (1993) Imaging vector fields using line integral convolution. In Proceedings of SIGGRAPH

31. Loring B, Karimabadi H, Rortershteyn V (2014) A screen space GPGPU surface LIC algorithm for distributed memory data parallel sort last rendering infrastructures. Tech. rep., Lawrence Berkeley National Lab.(LBNL), Berkeley, CA (United States)

32. Chong MS, Perry AE, Cantwell BJ (1990) A general classification of three-dimensional flow fields. Phys. Fluids A 2(5), 765-777

33. Hodzic E, Esmaeelzade G, Moshammer K, Fernandes R, Markus D, M. G., Early, J., and Grosshans, H., (2018) Analysis of the turbulent flow structure in a jet stirred reactor using proper orthogonal decomposition. IMEKO, Belfast, United Kingdom

34. Ayass WW (2013) Mixing in jet-stirred reactors with different geometries. Master's thesis, King Abdullah University of Science and Technology

35. Yeoh S, Papadakis G, Yianneskis M (2004) Numerical simulation of turbulent flow characteristics in a stirred vessel using the LES and RANS approaches with the sliding/deforming mesh methodology. Chem. Eng. Res. Des. 82(7), 834-848

36. Rasam A, Brethouwer G, Schlatter P, Li Q, Johansson AV (2011) Effects of modelling, resolution and anisotropy of subgrid-scales on large eddy simulations of channel flow. J. Turbul. 12(10), 1-19

37. Davidson L (2009) Large eddy simulations: How to evaluate resolution. Int. J. Heat Fluid Flow 30(5), 1016-1025

38. Sisemore C, Harvie JM, Skousen TJ (2016) Calculation of the dissipated energy spectrum from a fourier amplitude spectrum. Tech. rep., Sandia National Lab., United States

39. Grosshans H, Papalexandris MV (2017) Direct numerical simulation of triboelectric charging in particle-laden turbulent channel flows. J. Fluid Mech. 818:465-491

40. Kenney M, Sarjant R, Thring M (1956) The viscosity of mixtures of gases at high temperatures. Br. J. Appl. Phys. 7(9):324

41. Corrigan TE, Beavers WO (1968) Dead space interaction in continuous stirred tank reactors. Chem. Eng. Sci. 23(9), 1003-1006

Publisher's Note Springer Nature remains neutral with regard to jurisdictional claims in published maps and institutional affiliations. 